\title{
Urine Ammonium Biurate Crystal Measurement
}

National Cancer Institute

\section{Source}

National Cancer Institute. Urine Ammonium Biurate Crystal Measurement. NCI

Thesaurus. Code C74758.

The determination of the amount of ammonium biurate crystals present in a urine sample. 